\title{
Neurosurgical emergency management during the lockdown period in health care regions in Spain with different COVID-19 impact: lessons learned to improve outcomes on the future waves
}

\author{
Maria L. Gandía-González ${ }^{1}\left[\right.$. Jose M. Viñuela-Prieto ${ }^{1}$. Laura Barrios ${ }^{2} \cdot$ Carlos Alarcón $^{3} \cdot$ Fuat Arikan $^{4}$. \\ Cinta Arráez ${ }^{5}$. Carlos J. Domínguez ${ }^{6}$. Jose F. Alén ${ }^{7} \cdot$ Raquel Gutiérrez-González ${ }^{8,9} \cdot$ Angel Horcajadas $^{10}$. \\ Fernando Muñoz Hernández ${ }^{11}$. Alejandra Narváez ${ }^{12}$. Igor Paredes ${ }^{13} \cdot$ Rebeca Pérez-Alfayate $^{14}$. \\ Angel Rodríguez de Lope ${ }^{15} \cdot$ Fernando Ruiz-Juretschke $^{16} \cdot$ Freddy J. Salge Arrieta $^{17}$. Sonia Tejada ${ }^{18}$. \\ Martin Tamarit ${ }^{19} \cdot$ Thomaz Topczewski $^{20}$. Jesus Lafuente ${ }^{12}$
}

Received: 5 April 2021 / Accepted: 18 July 2021 / Published online: 16 August 2021

(c) Springer-Verlag GmbH Germany, part of Springer Nature 2021

\begin{abstract}
Background COVID-19 has overloaded health care systems, testing the capacity and response in every European region. Concerns were raised regarding the impact of resources' reorganization on certain emergency pathology management. The aim of the present study was to assess the impact of the outbreak (in terms of reduction of neurosurgical emergencies) during lockdown in different regions of Spain.

Methods We analyzed the impact of the outbreak in four different affected regions by descriptive statistics and univariate comparison with same period of two previous years. These regions differed in their incidence level (high/low) and in the time of excess mortality with respect to lockdown declaration. That allowed us to analyze their influence on the characteristics of neurosurgical emergencies registered for every region.

Results 1185 patients from 18 neurosurgical centers were included. Neurosurgical emergencies that underwent surgery dropped $24.41 \%$ and $28.15 \%$ in 2020 when compared with 2019 and 2018, respectively. A higher reduction was reported for the most affected regions by COVID-19. Non-traumatic spine experienced the most significant decrease in number of cases. Life-threatening conditions did not suffer a reduction in any health care region.

Conclusions COVID-19 affected dramatically the neurosurgical emergency management. The most significant reduction in neurosurgical emergencies occurred on those regions that were hit unexpectedly by the pandemic, as resources were focused on fighting the virus. As a consequence, life-threating and non-life-threatening conditions' mortality raised. Results in regions who had time to prepare for the hit were congruent with an organized and sensible neurosurgical decision-making.
\end{abstract}

Keywords Emergency $\cdot$ COVID-19 $\cdot$ Lockdown $\cdot$ Neurosurgery $\cdot$ Pandemic $\cdot$ Collateral damages

\section{Introduction}

The disruptive Severe Acute Respiratory Syndrome Coronavirus 2 (SARS-CoV2) pandemic tested our health care system resilience and preparedness, which was initially collapsed by a flood of COVID-19 patients being affected despite the measures that have been quickly implemented $[1,2]$.

Maria L. Gandía-González

marisagg4@hotmail.com

Extended author information available on the last page of the article
Attention has been inevitably directed towards COVID-19 patients and pandemic control efforts; therefore, healthcare reorganization at all levels (central state, autonomous communities and hospital centers) was indispensable to protect patients and staff and to increase the number of necessary resources to treat the high percentage of SARS-CoV2 infected patients $[3,4]$.

In our country, 3.733.600 COVID-19 cases have been confirmed to date 11th June 2021, and 80.501 deaths have been registered according to official registry by the Ministry of Health [5]. There were big differences in the incidence and time lapse between outbreak starting and planning, being Madrid, Castilla la Mancha, and Catalonia the 
most affected healthcare regions during the first outbreak, in this order [6]. Madrid started on March 8th, Castilla la Mancha, March 15th, and Catalonia on March 20th, considering the date of outbreak starting when excess mortality was registered by National Epidemiological Surveillance Network-Carlos III Institute [7].

The high incidence of COVID-19 patients in Spain has allowed us to build an important registry with information from hospitals in autonomous communities that experienced completely different scenarios. Due the decentralized management of the Spanish Public Health Care System, different approaches in terms of planification, organization, and strategy were applied in each autonomous community [4]. Some healthcare administrative regions were caught unprepared like Madrid that was severely hit without notice. Others, like Catalonia and Andalucía, where the outbreak was delayed by some days (12 and 13, respectively), were better organized and prepared for the pandemic.

Additionally, the message of generalized hospital collapse and the fear spread by mass media, dissuaded many patients to attend to the hospitals when feeling sick $[8,9]$. This situation led to a significant decrease in the number of emergencies related to pathologies different than COVID-19 [10,11].

The aim of the present study was to assess the impact of the outbreak (in terms of reduction of neurosurgical emergencies) during lockdown in different regions of Spain by comparing it with the same period of the 2 previous years. We undertook the analysis of the available data hoping that lessons learned might be useful to prevent excess of mortality and morbidity in patients for future waves of the SARSCoV-2 pandemic and be an example for other countries in need of health care reorganization.

\section{Methods}

\section{Design, setting, and population}

We retrospectively reviewed the database of the Neurosurgery Departments from 18 hospitals across Spain during the first outbreak. This study was approved by the coordinating center's Ethics Committee of Clinical Research and carried out following their recommendations.

Data from all neurosurgical emergencies registered between 14th March and 14th May 2020-lockdown period and peak of the wave-were retrospectively collected, including only patients $\geq 18$ years old who actually underwent surgery. In addition, neurosurgical emergencies that underwent surgery from the same period of time of the 2 previous years were also collected.

\section{Variables}

To explore the different regions across the country that suffered the strike of the pandemic at a different time we defined four categories. Briefly, the main epidemiological differences between them were incidence, time of outbreak declaration and whether the lockdown period had been declared or not when the outbreak started.

The groups were defined as follows:

-Group A (Madrid): high incidence, first region to achieve excess of mortality and this happened before lockdown (March 8th).

-Group B (Castilla la Mancha): high incidence, excess of mortality achieved 1 week later (March 15th), under lockdown.

-Group C (Catalonia): high incidence, excess of mortality achieved 12 days later (March 20th), under lockdown.

-Group D (Andalucía): low incidence, excess of mortality achieved 13 days later (March 21st), under lockdown.

Neurosurgical pathologies treated as emergency surgeries were classified into non-traumatic spine, trauma including traumatic brain injury (TBI) and traumatic spine, vascular, oncology, cerebrospinal fluid (CSF) related surgeries, and others (wound infection, functional, and miscellanea). Additionally, they were classified into life-threatening (trauma, vascular, oncology and CSF) and non-life-threatening pathologies (non-traumatic spine and others). Life-threatening conditions were considered those patients that required a surgical intervention to survive.

\section{Statistical analysis}

Descriptive statistics were expressed as mean \pm SD (standard deviation) for continuous variables, median (IQR; interquartile range) for ordinal variables and as $n(\%)$ for categorical variables. Comparison of proportions for categorical variables was performed by either Fisher's exact test or chi-squared test. For each value of the categorical variable, the rest of categories acted as reference.

To compare continuous variables among the three periods included in the study we used the ANOVA test with post hoc Bonferroni corrections for multiple comparisons. To compare ordinal variables and medians we employed the Kruskal-Wallis' H test. A Dunn-Bonferroni's nonparametric pairwise comparison for post hoc testing after a statistically significant Kruskal-Wallis test was also performed. The level of significance was defined as $P<0.05$.

All statistical analyses were performed using SPSS ${ }^{\circledR}$ Version 20 (IBM ${ }^{\circledR}$, Chicago, USA). 


\section{Results}

\section{Population and descriptive data}

We collected a total of 1185 patients from 18 centers who underwent an emergency neurosurgical procedure between March 14th and May 14th. Worth noting, a $24.41 \%$ and a $28.15 \%$ reduction in the number of patients were observed in 2020 when compared with 2019 and 2018, respectively. Results regarding distribution among different regions are summarized in Table 1. Patient and pathologies' characteristics are summarized in Table 2.

\section{Main results}

A chi-squared test was used to compare the number of surgeries between groups. All groups suffered a reduction in the volume of patients underwent surgery in 2020 in comparison with 2019 that ranged from a 39\% (group A) and $6 \%$ (group D) (Fig. 1). However, only groups A, B, and D reported a decrease in number of patients when comparing with 2018. Only group $\mathrm{C}$ showed a significant change in distribution $(P=0.012)$, with the lowest volume of patients in 2018 (Table 1).

No major redistribution of patients between hospitals within the same group was observed. Overall, all hospitals in group $\mathrm{A}$ and $\mathrm{B}$ experienced a wide decrease of neurosurgical emergencies. In group $\mathrm{C}$, all hospitals registered a reduced number of neurosurgical emergencies when compared with 2019. Group D showed a minimum reduction of cases.

Regarding patient characteristics, no significant differences were observed in age, gender, admission after the surgery, mean stay at ICU in days and mortality. Group C showed a statistically significant reduction on ward and ICU median stay.

We also evaluated the pandemic influence on the distribution of pathologies requiring an emergency operation during the study period (Figs. 2, 3). Non-traumatic spine operations were significantly reduced considering the whole sample and in group A, achieving statistical significance in

Table 1 Yearly and regionally distribution of neurosurgical emergencies

\begin{tabular}{lcccc}
\hline Group & 2018 & 2019 & 2020 & $P$ value \\
\hline Group A & $195(43.9)$ & $169(40)$ & $118(37)$ & 0.150 \\
Group B & $44(9.9)$ & $33(7.8)$ & $28(8.8)$ & 0.556 \\
Group C & $118(26.6)$ & $150(35.5)$ & $107(33.5)$ & 0.012 \\
Group D & $87(19.6)$ & $70(16.6)$ & $66(20.7)$ & 0.320 \\
Total & $444(100)$ & $422(100)$ & $319(100)$ & 0.083 \\
\hline
\end{tabular}

Results are expressed as $n(\%)$
2020. Trauma emergencies were significantly lower in 2020 in comparison with the previous years for the whole study sample. No statistically significant differences were detected among specific groups.

The proportion of vascular emergencies was not significantly different in 2020 with respect to previous years. Oncological emergency procedures experienced a significant decrease in group D in $2020(P<0.001)$, while in group $\mathrm{C}$ the tendency was the opposite $(P=0.027)$. CSF-related surgeries' frequency was significantly higher among the whole sample and particularly in group A $(P<0.001$ and $P=0.001$, respectively).

Regarding the COVID-19's PCR results obtained from patients attended in 2020, an overall $7.5 \%$ positive rate was observed. Among groups, the highest positive rate was observed in group B and the lowest in group D. The amount of indeterminate results ranged from $0 \%$ in group $\mathrm{B}$ to as high as a $29 \%$ in group C. Importantly, the rate of negativeto-positive change during stay was not negligible for certain groups, such as A and B, where it was above 5\% (Table 3).

Regarding the median GCS at their arrival at the ER, only a significant reduction was observed when comparing 2020 to the previous years in groups $\mathrm{A}$ and $\mathrm{D}$. When comparing GCS at arrival between life-threatening and non-life-threatening pathologies, no statistically significant differences were observed among the 3 years studied. When this comparison was further explored for each group no significant differences were observed whatsoever (Table 4).

The proportion of emergency neurosurgical patients admitted in ICU beds increased significantly in 2020 when compared with previous years for all groups. Groups A, B and $C$ reported a higher increase than group D from 2019 to 2020, without statistical significance though. In terms of ICU stay, the mean stay was reduced, although not significantly, in groups A, B, and C, but not in group D. Interestingly, when considering the median ICU stay, the reduction of the median ICU stay was significant $(P=0.015)$ when comparing 2019 with $2020(P=0.019)$ in group $C$. The same tendency among groups was observed regarding the mean and the median global stay, being reduced in all groups but achieving statistical significance only in group $\mathrm{C}(P=0.025$ and $P<0.001$, respectively).

Mortality analysis showed a worrying, yet not significant, increase of mortality for our emergency neurosurgical operations in all groups when comparing 2020 with either 2019 or 2018. In fact, group A alone experienced a 6-7\% increased mortality when compared with previous years (Table 5).

Mortality rates for non-life-threatening pathologies were significantly higher in 2020 whereas mortality rates for lifethreatening pathologies were not. Further analysis regarding the pathologies registering higher rates of mortality during 2020 revealed that functional, infection-related, and other 


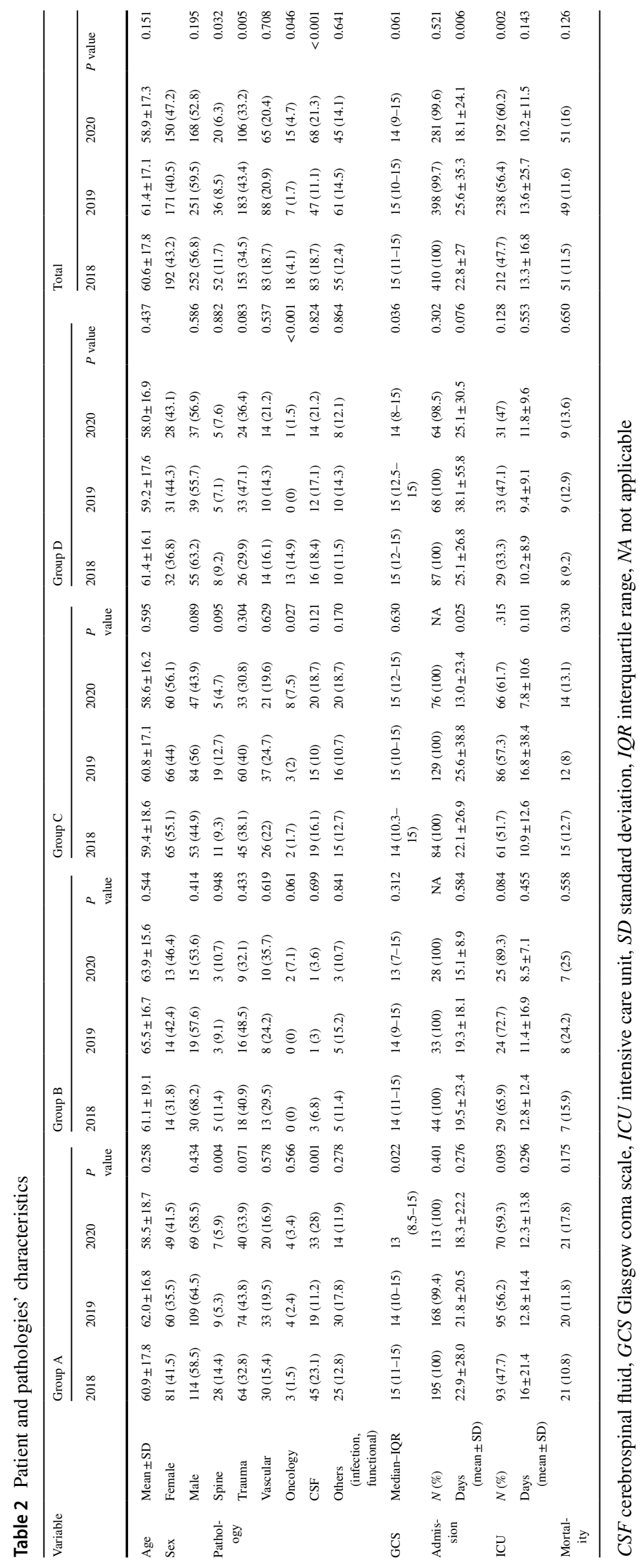


Fig. 1 Neurosurgical emergencies' evolution during study time. Arrows represent percentual variation with respect to previous years

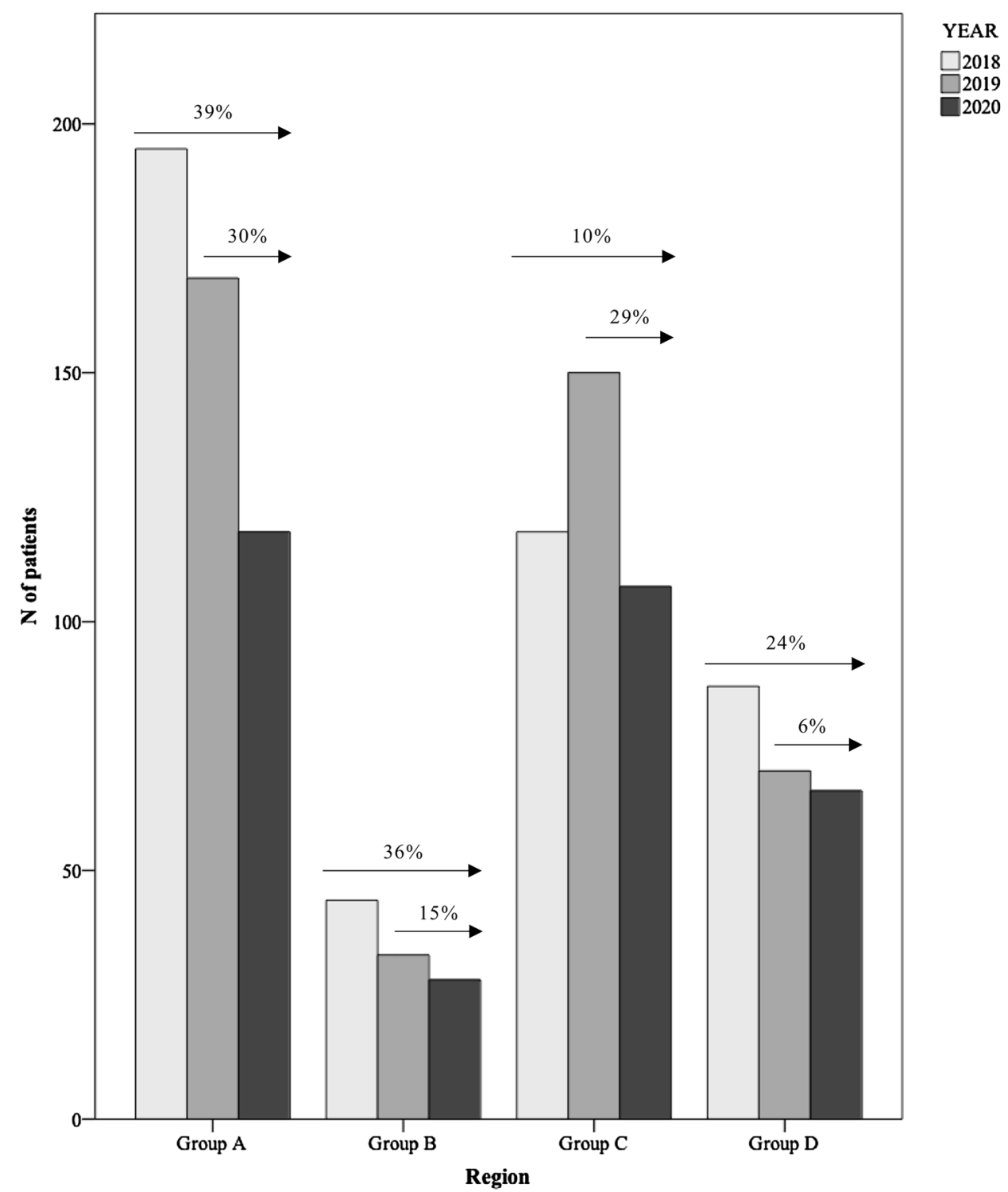

pathologies were the only ones experiencing a significant increase during 2020.

\section{Discussion}

COVID-19 pandemic has changed our paradigm of surgical care, testing our capacity to prioritize one patient over another and to develop decision-making processes that ensure an adequate management to our patients. Concern about neurosurgical emergencies and potential "collateral damages" has been raised among neurosurgeons throughout the world [5]. Reallocation of resources and medical staff to the COVID-19 patient's has hugely affected neurosurgical patient care delivery, particularly in severely affected countries such as Spain $[1,4]$. Not only the number of available neurosurgical ward and Intensive Care
Unit (ICU) beds were highly reduced in many European countries [12], but many of staff neurosurgeons were also relocated to Emergency and Internal Medicine Departments [13] while elective surgeries were put on hold in the most hit countries for a long period of time [14, 15].

On these grounds, we analyzed the different impact of COVID-19 pandemic on neurosurgical emergency procedures based on the preparation and response capacity. To do so, we compared the volume and characteristics with those from emergencies attended during the same period of the two previous years, 2018 and 2019. Other similar studies conducted in other countries have included variable time frames as controls, often considering only the year before [16-18]. Due to the intrinsic variability of neurosurgical emergencies along time, we considered that including a longer period of time for comparison would add further validity to our conclusions. 


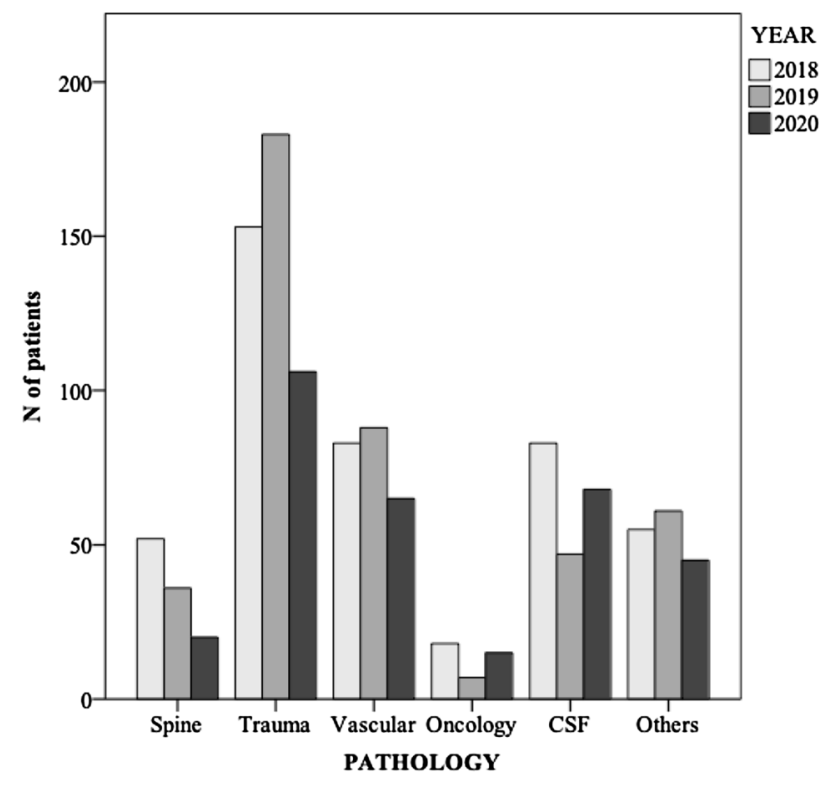

Fig. 2 Emergency surgeries for every pathology evolution during study time. CSF cerebrospinal fluid-related pathologies

Furthermore, resembling the impact of an earthquake, we considered 4 different regions along the Spanish geography (Fig. 4). Other single-country studies have tried to longitudinally analyze the impact of the pandemic by setting a timeline along the 2020's March-May period [19]. Despite the restricted national framework of the present study, we consider that this subdivision based on the pandemic timing widens its international applicability.

Inside our borders a broad spectrum of situations regarding incidence, health care saturation and excess of mortality were seen depending on the region considered, as our health care management and organization is transferred to regional political powers.

Trias-Llimós et al [4]. Previously expressed their concerns about poor coordination among central and regional authorities and slow decision-making processes in some cases, showing the weakness of a fragile decentralized system. They urged to analyze the situation in different regions to identify health care needs that might be improved for the following waves.

In our study, we analyzed four different time windows based on the excess of mortality instead of the first positive cases, since that was the actual parameter that preceded health care overrun and precluded the most drastic confinement measures. Other similar studies have only considered a single time frame based on the identification of the first COVID-19 cases [18].

Globally, the data showed a marked decrease in the total number of procedures performed during the same period this year 2020. This is consistent with the majority of studies assessing the repercussion of COVID-19 on neurosurgical activity [17, 18, 20-24]. Conversely, other authors have reported an increase in the neurosurgical hospitalization and treatment of their respective centers [16]. Those particular cases were usually observed in the context of emergency network reorganization, where hub hospitals were focused on the management of non-COVID-19 pathologies, a clear example of a better organization.

The percentual distribution of cases among the four groups did not significantly varied along time, with the exception of group $\mathrm{C}$ that raised from a $26.6 \%$ of cases in 2018 to a $35.5 \%$ in 2019 and a $33.5 \%$ in 2020 . That exception could be explained due to the relatively maintained number of surgeries in that group that had enough time to prepare for the outbreak noting a smaller reduction in number of emergencies in the context of a marked reduction of patients in groups A and B.

We classified every neurosurgical emergency into one out of six different categories. In that sense, we share many classification criteria with other similar studies published to date $[18,23]$.

Nonetheless, in our work, we distinguished between degenerative and traumatic spinal injuries, including each of them in a separated category (spine and trauma, respectively). We considered that, despite sharing the anatomical location, both types of pathologies are sufficiently different by means of acuity of onset, patient profile, global severity of the associated lesions, medical and extra-hospital management to get classified in different categories. Additionally, we considered traumatic spine caseload had probably dropped due to lockdown, but there was no reason to record a reduction of the rate of non-traumatic spine emergencies.

Regarding the type of pathology attended during the 2020 lockdown period, it is worth noting that at the epicenter of the pandemic, namely group A, the number of non-traumatic spine on-call surgeries significantly dropped. These observations have also been reported by other authors $[17,20]$. Probably, the deterrent effect of the pandemic on the ER frequentation, the patients' fear and the cancellation of consultations may have accounted for this finding. In this regard, we consider that this type of pathology should be monitored, either by remote consultation or telemedicine, in the yet-tocome waves of the pandemic to avoid complications derived from more advanced cases.

For every period of time considered in the present study, traumatic injuries were the most frequent causes to perform emergency surgery. This finding is shared by other authors $[18,24]$. Nevertheless, trauma emergencies were significantly lower in 2020 in comparison with 2019 for the whole sample. Those results could be explained by both the reduction of road mobility and the closure of work centers associated to the lockdown state. 
Fig. 3 Non-life-threatening and life-threatening emergency surgeries during study time (expressed as a percentage of total emergency surgeries). Mortality percentage evolution during study time for non-lifethreatening and threatening pathologies

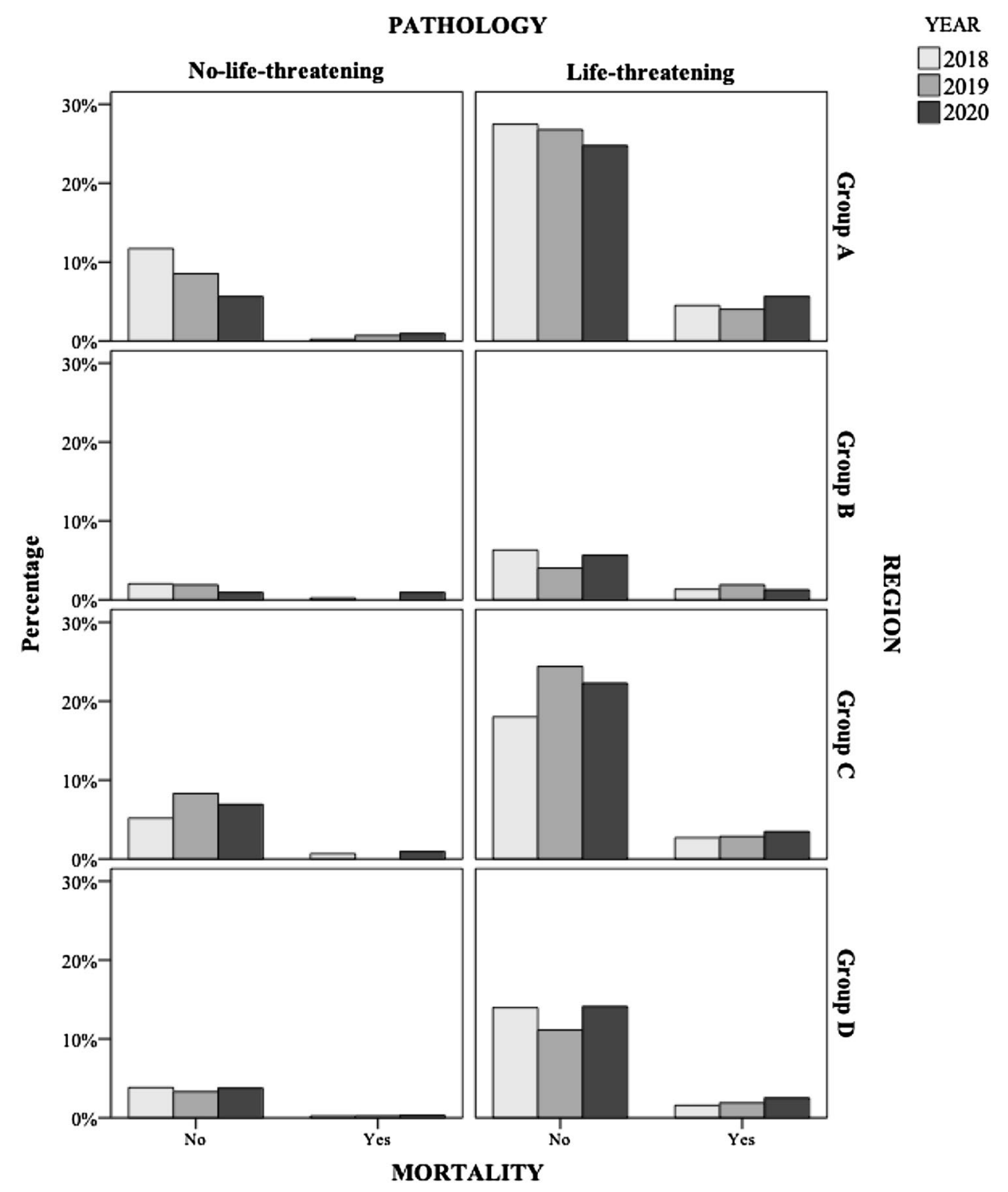

Conversely to the generalized reduction observed in other types of neurosurgical emergencies, oncological and CSFrelated surgeries experienced a proportional increase. Other similar studies have also identified this tendency, attributing it to prioritization policies during the pandemic [17, 18, 21], as due to cancelled elective-surgery and consultations, emergent surgeries drifted to either more severe, impossible-topostpone and were operated by the emergency team. However, the fact that the absolute numbers remain relatively similar to previous years may indicate that the proportional increase is due to the reduction of the rest of pathologies.

In our study, the mean and the median GCS obtained at Emergency Room (ER) arrival did not significantly varied, with the exception of the most and the least pandemicaffected groups. Nevertheless, when differentiating between life-threatening and non-life-threatening pathologies those differences were not significant for both groups. Other studies have also identified this reduction in GCS during the pandemic in vascular emergencies, hydrocephalus and traumatic brain injury [18].

The proportion of patients needing an ICU bed after the surgery suffered an increase in all groups. That shows a good and responsible decision-making for patients, operating only those that have life-threatening conditions. However, in exchange, the non-life-threatening conditions may have been left behind and, consequently, would have suffered an increase in mortality, particularly in group A.

If we consider these data, we could conclude that group $\mathrm{C}$ had enough time to prepare the resources and optimizing the turnover of the ICU beds. Also, these centers applied less ICU-time-consuming procedures that could be managed 
Fig. 4 Geographical categorization into four different groups depending on percentage and date of excess mortality, resembling the impact of an earthquake

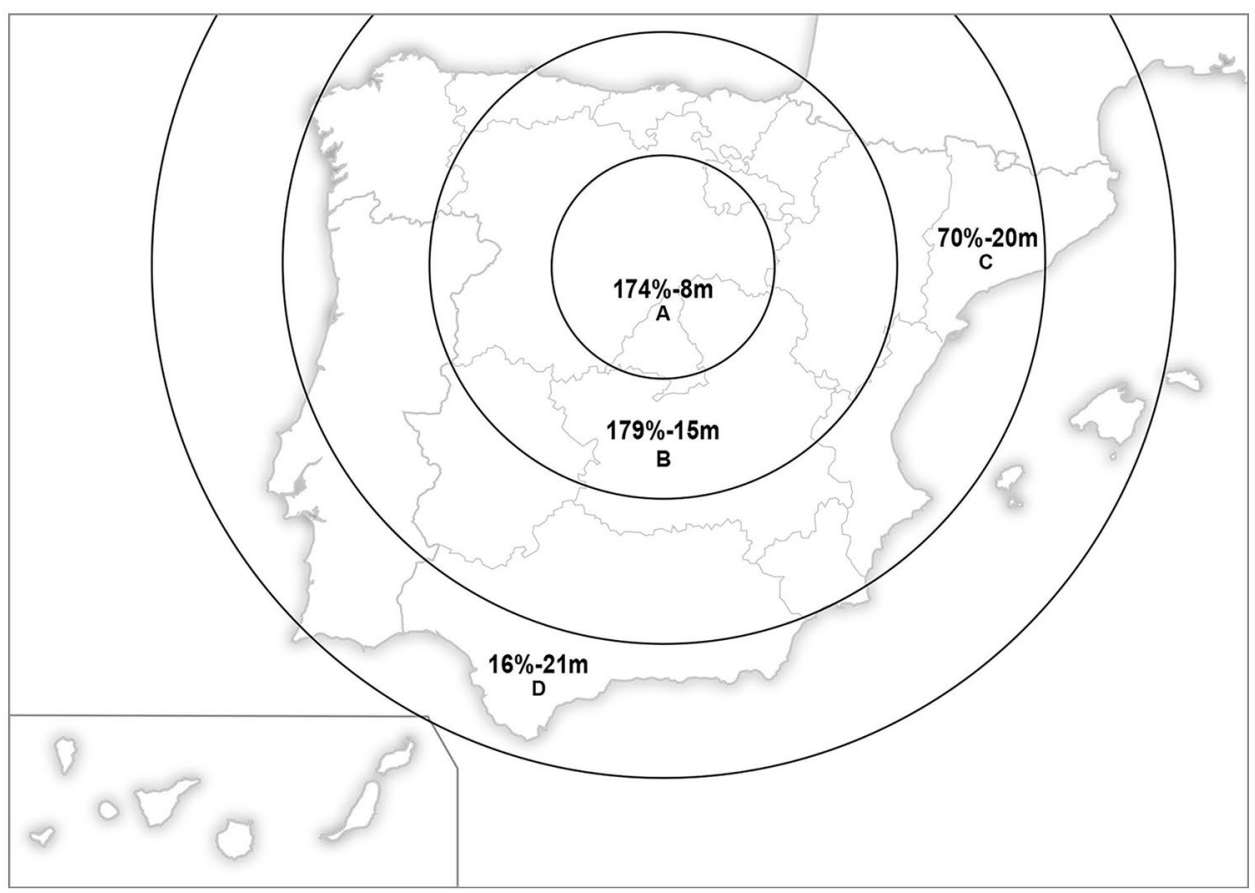

Table 3 COVID-19's PCR results

\begin{tabular}{lcccc}
\hline & \multicolumn{2}{l}{$2020(N=319)$} & & \\
\cline { 2 - 5 } & Indeterminate & Negative & Positive & $\begin{array}{l}\text { Positivized } \\
\text { during stay }\end{array}$ \\
\hline Group A & $3(2.5)$ & $95(80.5)$ & $14(11.9)$ & $6(5.1)$ \\
Group B & $0(0)$ & $19(67.9)$ & $6(21.4)$ & $3(10.7)$ \\
Group C & $31(29)$ & $71(66.4)$ & $4(3.7)$ & $1(0.9)$ \\
Group D & $8(12.1)$ & $56(84.8)$ & $0(0)$ & $2(3)$ \\
Total & $42(13.2)$ & $241(75.5)$ & $24(7.5)$ & $12(3.8)$ \\
\hline
\end{tabular}

Results are expressed as $n(\%)$

Table 4 Yearly distribution of GCS depending on the severity of the pathology

\begin{tabular}{lllll}
\hline & \multicolumn{4}{l}{ GCS } \\
\cline { 2 - 5 } & 2018 & 2019 & 2020 & $P$ value \\
\hline Non-life-threatening & $15(15-15)$ & $15(15-15)$ & $15(13-15)$ & 0.137 \\
Life-threatening & $14(9-15)$ & $14(9-15)$ & $13(8-15)$ & 0.397 \\
\hline
\end{tabular}

Results are expressed as median (IQR)

with a short ICU stay to be performed during the peak of the wave.

In our study, mortality did not significantly increased during the 2020 lockdown with respect to the previous years. This finding is in line with other similar studies evaluating the impact of COVID-19 on neurosurgical practice [17].
Table 5 Yearly distribution of mortality regarding severity of the pathology

\begin{tabular}{lccll}
\hline & \multicolumn{3}{c}{ Mortality } & \\
\cline { 2 - 5 } & 2018 & 2019 & 2020 & $P$ value \\
\hline Non-life-threatening & $6(5.6)$ & $4(4.1)$ & $10(15.4)$ & 0.018 \\
Life-threatening & $45(13.4)$ & $45(13.8)$ & $41(16.1)$ & 0.605 \\
\hline
\end{tabular}

Results are expressed as $n(\%)$

Mortality did not seem to significantly differ in groups C and $\mathrm{D}$ with respect to previous years. This supports that the fact that $\mathrm{C}$ and $\mathrm{D}$ did have more time to anticipate to the outbreak hospital crisis has an influence on patients' outcomes.

Nevertheless, in group A, a 6-7\% increase in mortality was observed in comparison with 2018 and 2019, respectively. Regardless of statistical significance, we believe that those numbers could not be unapprised.

All the patients included in our sample were tested for SARS-CoV2 infection preoperatively by means of PCR test. Globally, a 7.5\% positive rate was detected. Nevertheless, positive rates widely varied among regions (from $0 \%$ to $21.4 \%$ ), being higher in the most affected ones. The fact that mortality was not significantly higher in 2020 when compared to previous years suggests that, at least in our sample, SARS-CoV2 infection did not add further risk to neurosurgical procedures.

The interpretation of the present study has some limitations. First, groups $\mathrm{A}$ and $\mathrm{C}$ represented big population 
groups with 482 and 375 patients included, respectively. However, group B only included 105 patients which makes it difficult to draw conclusions for that group.

The retrospective nature of the present study, based on database undertaken after the outbreak, represents a limitation that is not uncommon in other studies evaluating the effects of the pandemic on habitual medical or surgical practice. Nevertheless, and despite the difficulty in predicting subsequent strikes of COVID-19, prospective studies monitoring the impact over the neurosurgical activity and its derived mortality and morbidity should be taken into account for future investigations.

Furthermore, although data were collected from some of the busiest centers across the country, not every center in our country was included.

\section{Conclusions}

COVID-19 affected dramatically the neurosurgical emergency management. The most significant reduction in neurosurgical emergencies occurred on those regions that were hit unexpectedly by the pandemic, as resources were focused on fighting the virus (putting on hold elective surgeries either in prevision or in need of reorganization of the ICUs). As a consequence, life-threating and non-life-threatening conditions' mortality raised. Results in regions who had time to prepare for the hit were congruent with an organized and sensible neurosurgical decision-making. Although things could had been better managed in Spain both locally and globally, we need to keep looking forward and learn how to address potential health threats far in advance to avoid chaos and unexpected death.

\section{Declarations}

Conflict of interest The authors declare that there are no conflict of interests.

\section{References}

1. The Lancet Public Health. COVID-19 in Spain: a predictable storm? Lancet Public Health. 2020;5(11):e568.

2. Legido-Quigley H, Mateos-García JT, Campos VR, Gea-Sánchez M, Muntaner C, McKee M. The resilience of the Spanish health system against the COVID-19 pandemic. Lancet Public Health. 2020;5(5):e251-2.

3. Meschi T, Rossi S, Volpi A, Ferrari C, Sverzellati N, Brianti E, et al. Reorganization of a large academic hospital to face COVID-19 outbreak: the model of Parma, Emilia-Romagna region Italy. Eur J Clin Invest. 2020;50(6):e13250. https://doi.org/10.1111/eci.13250.

4. Trias-Llimós S, Alustiza A, Prats C, Tobias A, Riffe T. The need for detailed COVID-19 data in Spain. Lancet Public Health. 2020;5(11):e576. https://doi.org/10.1016/S2468-2667(20)30234-6.

5. Centro de Coordinación de Alertas y Emergencias Sanitarias. Ministerio de Sanidad. Actualización $n^{\circ} 395$. Enfermedad por el coronavirus (COVID-19). 11.06.2021. https://www.mscbs.gob.es/profe
sionales/saludPublica/ccayes/alertasActual/nCov/documentos/Actua lizacion_395_COVID-19.pdf. Accessed 14 June 2021.

6. Henríquez J, Gonzalo-Almorox E, García-Goñi M, Paolucci F. The first months of the COVID-19 pandemic in Spain. Health Policy Technol. 2020;9(4):560-74.

7. Red Nacional de Vigilancia Epidemiológica. Instituto de Salud Carlos III. Ministerio de Ciencia e Innovación. https://eng.isciii.es/eng. isciii.es/Paginas/Inicio.html. Accessed 14 June 2021.

8. McFadden SM, Malik AA, Aguolu OG, Willebrand KS, Omer SB. Perceptions of the adult US population regarding the novel coronavirus outbreak. PLoS ONE. 2020. https://doi.org/10.1371/journal. pone. 0231808 .

9. Tartara F, Cofano F, Zenga F, Boeris D, Garbossa D, Cenzato M. Are we forgetting non-COVID-19-related diseases during lockdown? Acta Neurochir (Wien). 2020;162(7):1501.

10. Hammad TA, Parikh M, Tashtish N, Lowry CM, Gorbey D, Forouzandeh F, et al. Impact of COVID-19 pandemic on ST-elevation myocardial infarction in a non-COVID-19 epicenter. Catheter Cardiovasc Interv. 2020;97(2):208-14.

11. Lange SJ, Ritchey MD, Goodman AB, et al. Potential indirect effects of the COVID-19 pandemic on use of emergency departments for acute life-threatening conditions-United States, January-May 2020. MMWR Morb Mortal Wkly Rep. 2020;69:795-800.

12. Mathiesen T, Arraez M, Asser T, Balak N, Barazi S, Bernucci C, et al. EANS Ethico-legal committee. A snapshot of European neurosurgery December 2019 vs. March 2020: just before and during the Covid-19 pandemic. Acta Neurochir (Wien). 2020;162(9):2221-33.

13. Gandía-González ML, Sáez-Alegre M, Roda JM. Neurosurgeons on the frontline of COVID-19: no place for surgery? Acta Neurochir (Wien). 2020;162(7):1503-4.

14. Huang Z, Zhao S, Li Z, Chen W, Zhao L, Deng L, et al. The battle against coronavirus disease 2019 (COVID-19): emergency management and infection control in a radiology department. J Am Coll Radiol. 2020;17(6):710-6.

15. Hulsbergen AFC, Eijkholt MM, Balak N, Brennum J, Bolger C, Bohrer AM, et al. Ethical triage during the COVID-19 pandemic: a toolkit for neurosurgical resource allocation. Acta Neurochir (Wien). 2020;162(7):1485-90.

16. Agosti E, Giorgianni A, Pradella R, Locatelli D. Coronavirus disease 2019 (COVID-19) outbreak: single-center experience in neurosurgical and neuroradiologic emergency network tailoring. World Neurosurg. 2020;138:548-50.

17. Bajunaid K, Alqurashi A, Alatar A, Alkutbi M, Alzahrani AH, Sabbagh AJ, et al. Neurosurgical procedures and safety during the COVID-19 Pandemic: a case-control multicenter study. World Neurosurg. 2020;S1878-8750(20):31614-24.

18. Hecht N, Wessels L, Werft FO, Schneider UC, Czabanka M, Vajkoczy P. Need for ensuring care for neuro-emergencies-lessons learned from the COVID-19 pandemic. Acta Neurochir (Wien). 2020;162(8):1795-801.

19. Bajunaid K, Alatar A, Alqurashi A, Alkutbi M, Alzahrani AH, Sabbagh AJ, et al. The longitudinal impact of COVID-19 pandemic on neurosurgical practice. Clin Neurol Neurosurg. 2020. https://doi.org/ 10.1016/j.clineuro.2020.106237.

20. El-Ghandour NMF, Elsebaie EH, Salem AA, Alkhamees AF, Zaazoue MA, Fouda MA, et al. Letter: the impact of the coronavirus (COVID-19) pandemic on neurosurgeons worldwide. Neurosurgery. 2020;87(2):E250-7.

21. Jean WC, Ironside NT, Sack KD, Felbaum DR, Syed HR. The impact of COVID-19 on neurosurgeons and the strategy for triaging non-emergent operations: a global neurosurgery study. Acta Neurochir (Wien). 2020;162(6):1229-40.

22. Krenzlin H, Bettag C, Rohde V, Ringel F, Keric N. Involuntary ambulatory triage during the COVID-19 pandemic - a neurosurgical perspective. PLoS ONE. 2020. https://doi.org/10.1371/journal. pone.0234956. 
23. Sivakanthan S, Pan J, Kim L, Ellenbogen R, Saigal R. Economic impact of COVID-19 on a high-volume academic neurosurgical practice. World Neurosurg. 2020;S1878-8750(20):31792-7.

24. Soriano Sánchez JA, Perilla Cepeda TA, Zenteno M, Campero A, Yampolsky C, Varela ML, et al. Early report on the impact of
COVID-19 outbreak in neurosurgical practice among members of the Latin American Federation of Neurosurgical Societies. World Neurosurg. 2020;140:e195-202.

\section{Authors and Affiliations}

\section{Maria L. Gandía-González ${ }^{1}$ (D) . Jose M. Viñuela-Prieto ${ }^{1} \cdot$ Laura Barrios $^{2} \cdot$ Carlos Alarcón $^{3} \cdot$ Fuat Arikan $^{4}$. Cinta Arráez ${ }^{5}$. Carlos J. Domínguez ${ }^{6}$ · Jose F. Alén ${ }^{7} \cdot$ Raquel Gutiérrez-González $^{8,9} \cdot$ Angel Horcajadas $^{10}$. Fernando Muñoz Hernández ${ }^{11}$. Alejandra Narváez ${ }^{12} \cdot$ Igor Paredes $^{13} \cdot$ Rebeca Pérez-Alfayate $^{14}$. Angel Rodríguez de Lope ${ }^{15} \cdot$ Fernando Ruiz-Juretschke $^{16} \cdot$ Freddy J. Salge Arrieta $^{17} \cdot$ Sonia Tejada $^{18}$. Martin Tamarit ${ }^{19} \cdot$ Thomaz Topczewski $^{20} \cdot$ Jesus Lafuente ${ }^{12}$}

1 Department of Neurosurgery, La Paz University Hospital, Idipaz, Paseo de La Castellana, 261, 28046 Madrid, Spain

2 Department of Statistics CSIC, Madrid, Spain

3 Department of Neurosurgery, Hospital de Terrassa, Terrassa, Spain

4 Department of Neurosurgery, Neurotraumatology and Neurosurgery Research Unit (UNINN), Vall d'Hebron University Hospital and Vall d'Hebron Research Institute, Barcelona, Spain

5 Department of Neurosurgery, Carlos Haya University Hospital, Málaga, Spain

6 Department of Neurological Surgery, Germans Trias i Pujol University Hospital, Badalona, Spain

7 Department of Neurosurgery, La Princesa University Hospital, Madrid, Spain

8 Department of Neurosurgery, Puerta de Hierro University Hospital, Madrid, Spain

9 Department of Surgery, Faculty of Medicine, Autonomous University of Madrid, Madrid, Spain

10 Neurosurgery Department, Virgen de las Nieves University Hospital, Granada, Spain
11 Neurosurgery, Hospital de la Santa Creu i Sant Pau, Autonomous University of Barcelona, Barcelona, Spain

12 Department of Neurosurgery, Parc de Salut Mar, Barcelona, Spain

13 Neurosurgery Department, Hospital 12 de Octubre, Madrid, Spain

14 Department of Neurological Surgery, Hospital Clínico San Carlos, Madrid, Spain

15 Department of Neurosurgery, Virgen de la Salud Hospital, Toledo, Spain

16 Department of Neurosurgery, Hospital General Universitario Gregorio Marañón, Madrid, Spain

17 Department of Neurosurgery, Ramón y Cajal University Hospital, Madrid, Spain

18 Department of Neurosurgery, Fundación Jiménez Díaz (IIS-FJD), Madrid, Spain

19 Department of Neurosurgery, Hospital Universitario de Getafe, Getafe, Spain

20 Department of Neurological Surgery, Hospital Clinic, Barcelona, Spain 